\title{
Vademecum-based GFEM (V-GFEM): Optimal Enrichment for transient problems
}

\author{
Diego Canales $^{1, *}$, Adrien Leygue $^{1}$, Francisco Chinesta $^{1}$, David González $^{2}$, Elías Cueto $^{2}$, \\ Eric Feulvarch ${ }^{3}$, Jean-Michel Bergheau ${ }^{3}$, and Antonio Huerta ${ }^{4}$ \\ ${ }^{1}$ École Centrale de Nantes, France. \\ ${ }^{2}$ Aragón Institute of Engineering Research (I3A), Universidad de Zaragoza, Spain. \\ ${ }^{3}$ University of Lyon, ENISE, LTDS, UMR 5513 CNRS, Saint-Etienne, France. \\ ${ }^{4}$ Laboratori de Càlcul Numèric (LaCaN), Universitat Politecnica de Catalunya·BarcelonaTech, Barcelona, Spain.
}

\begin{abstract}
SUMMARY
This paper proposes a Generalized Finite Element Method based on the use of parametric solutions as enrichment functions. These parametric solutions are precomputed off-line and stored in memory in the form of a computational vademecum so that they can be used on-line with negligible cost. This renders a more efficient computational method than traditional Finite Element Methods (FEM) at performing simulations of processes. One key issue of the proposed method is the efficient computation of the parametric enrichments. These are computed and efficiently stored in memory by employing Proper Generalized Decompositions (PGD). Although the presented method can be broadly applied, it is particularly well suited in manufacturing processes involving localized physics which depend on many parameters, such as welding. After introducing the V-GFEM formulation, we present some numerical examples related to the simulation of thermal models encountered in welding processes.
\end{abstract}

Received ...

KEY WORDS: Vademecum-based Generalized Finite Element Method; Generalized Finite Element Method; Proper Generalized Decomposition; Transient Problem; Welding Simulation.

\section{INTRODUCTION}

Currently, the most advanced industries such as those involved with aerospace, automotive, nuclear, naval or energy technologies demand computer simulations of complex manufacturing processes. These simulations can save both time and resources in designing and industrial manufacturing due to the "in-silico" optimization of the process. Thus, slow and costly test campaigns can be avoided and the trial and error approach is significantly minimized.

*Correspondence to: Diego Canales. École Centrale de Nantes, 1 rue de la Noë, F-44321 Nantes, France. e-mail: diego.canales-aguileradec-nantes. fr

Contract/grant sponsor: ESI group and Spanish Ministry of Economy and Competitiveness; contract/grant number: Centrale Nantes - ESI Chair and CICYT DPI2014-51844-C2-R and 51844-C2-2-R 
Numerical simulation makes it possible to obtain variables that are difficult to observe experimentally and at a much lower cost than in a test campaign. Because of this, they can be integrated into the earliest stages of product design. In other words, today's advanced simulations have the potential to optimize the entire production cycle like never before. It is not surprising that its development is a strategic objective for industries and governments [1].

However, most manufacturing processes are very complex and involve a large number of physical phenomena that must be suitably modeled. The simulation of welding, one of the processes that has aroused the most interest in the industry and in many research groups [2] is a good example of this. If one analyzes the state-of-the-art simulation codes, the interaction of the electrode and the workpiece can be simulated within an electromagnetic model, the thermal model can be coupled with a metallurgical one, and finally, the whole thermal history can interact with an elasto-plastic mechanical model.

Even if one assumes that we have sufficient physical models that accurately represent reality, classical computational approaches are usually unworkable due to the demands they place on both resources and time.

Model Order Reduction (MOR) has helped formulate responses to this challenge in recent years [3-6], not only providing very powerful methods, but reformulating classical approaches to significantly improve their computational efficiency. This is precisely the objective of this work.

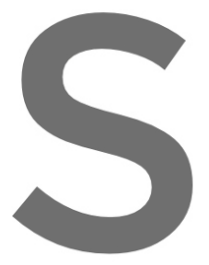
Within this realm, one could think of an ideal simulation meth
proyiding results at very high fredback rates, and is also capable
Undoubtedly, the Generalized Finite Element Method (GFEM) [1
this description well. By using enrichmen functions. GFEM is able
subgrid features of the physics underneath. The main difficulty ass the development of an efficient and accurate strategy to compute these enrichment functions.

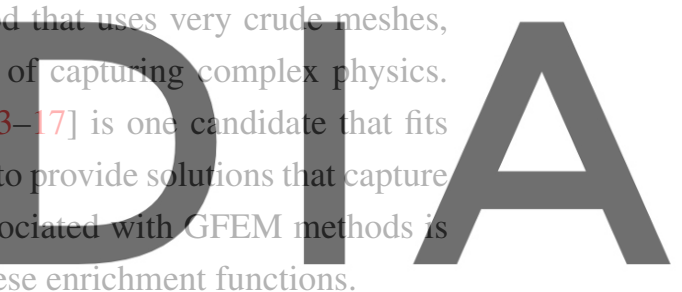
Register for free athttps/WwWW.Scipedia.com to download the version without the watermark can hardly serve for the enrichment of every element in the mesh, regardless of the physics at that particular region.

For this reason, the main objective of the work presented here is the development of a method which is able to capture sharp features of the solution, in a variety of different scenarios, with minimal extra degrees of freedom. To obtain such an ideal enrichment function, one can imagine a sort of parametric function dependent on boundary conditions, material characteristics, load values and other parameters that could be particularized to fit the approximation space at every region of the model.

Obtaining such a solution is of course not an easy task. However, computational techniques exist which are capable of obtaining such response surfaces efficiently. Among these techniques, Proper Generalized Decompositions (PGD) [5] is particularly attractive, due to its ability to efficiently construct the aforementioned parametric functions.

The resulting method, which we have called Vademecum-GFEM (V-GFEM), is no more than a finite element method that introduces, transparently to the user, an enrichment consisting of just one single function.

V-GFEM is a general-purpose method. However, it is particularly well adapted to transient problems involving localized physics dependent on several technological parameters. This is the 
case, for example, of welding and surface heat treatments. In this paper, the V-GFEM formulation and some numerical examples related to the mentioned processes are presented.

After this introduction, the paper is organized as follows. In Section 2, GFEM is presented as well as the major approaches that exist in the literature to introduce the enrichment functions, a crucial issue in this method. In Section 3, the V-GFEM is presented, the adaptative enrichment using the Computational Vademecum à la PGD is explained, giving the main ideas underpinning its construction. In this section, details about its implementation are also included. In Section 4 , the suitability of the V-GFEM for transient problems is presented through several numerical examples. Finally, in Section 5, we present our conclusions and discuss developments and future applications of the V-GFEM.

\section{GENERALIZED FINITE ELEMENT METHOD}

The accuracy obtained with a numerical method, and in particular with FEM, largely depends on how suitably the method has been adapted to the specific problem. In practice, we can follow different strategies to adapt our FEM formulation to the problem at hand. Some traditional strategies are $h$-adaptivity, $p$-adaptivity or $h p$-adaptivity [7]. These classical strategies are implemented in most

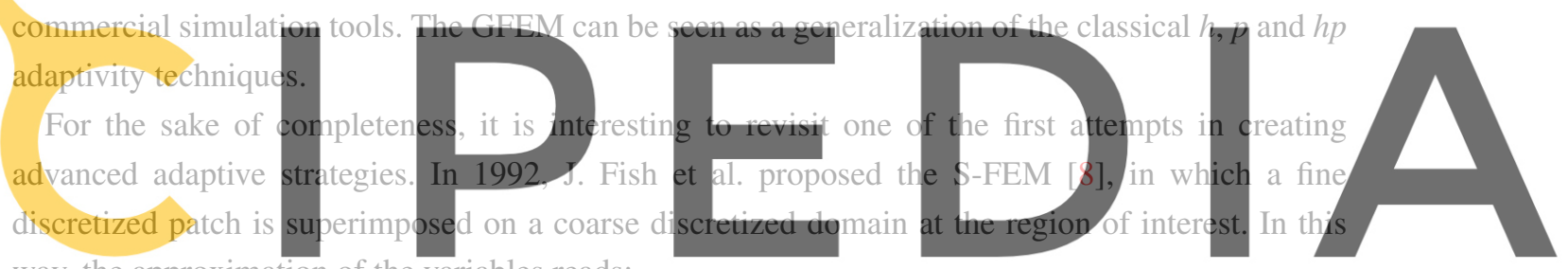
way, the approximation of the variables reads:

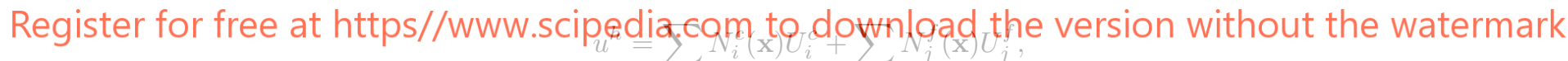

where one can see two different kinds of shape functions: the set of $N^{c}(\mathbf{x})$ functions associated to the coarse mesh (global domain) and the set of $N^{f}(\mathbf{x})$ functions associated to the fine one (patch). The continuity between the patch and the global domain can be ensured imposing $U^{f}=0$ at the boundary of the patch. In this manner, S-FEM tries to avoid remeshing, a real bottleneck in 3D simulations. However, some precautions should be taken to handle two computational meshes [9]:

- Numerical quadratures are in general difficult to perform. A special mesh (intersection of the coarse and the fine one) should be created to achieve accurate results.

- Rank deficiency must be prevented.

We have applied this technique in [10], [11] and [12] for locally-enriching solutions expressed in a reduced basis.

Some years later, the Generalized Finite Element Method (GFEM) was proposed by Melenk and Babuška [13]. The main idea of this method is to introduce in the trial space the available information about the solution.

There are many other techniques based on the same idea such as PUM, PUFEM, XFEM or Special FEM [14-20]. The GFEM is based in two properties: 
- Local approximability. GFEM is able to construct space of functions which can approximate the solution even better than the FE space of piece-wise polynomials.

- Conformity. The method is able to preserve the inter-element continuity without losing the approximation properties.

The first property will depend on the suitability of the so-called enrichment functions for a given problem. This question will be discussed in detail in subsection 2.1 because it is a key aspect of this work. With respect to the second one, the inter-element continuity is guaranteed if the enrichment is introduced in a space which satisfies the partition of unity. The formal definition of partition of unity can be found in [13], and it can be used to construct these spaces in a general manner, even without a mesh. In our context it is enough to say that in any traditional finite element discretization of a domain $\Omega$, the trial space satisfies the partition of unity if and only if:

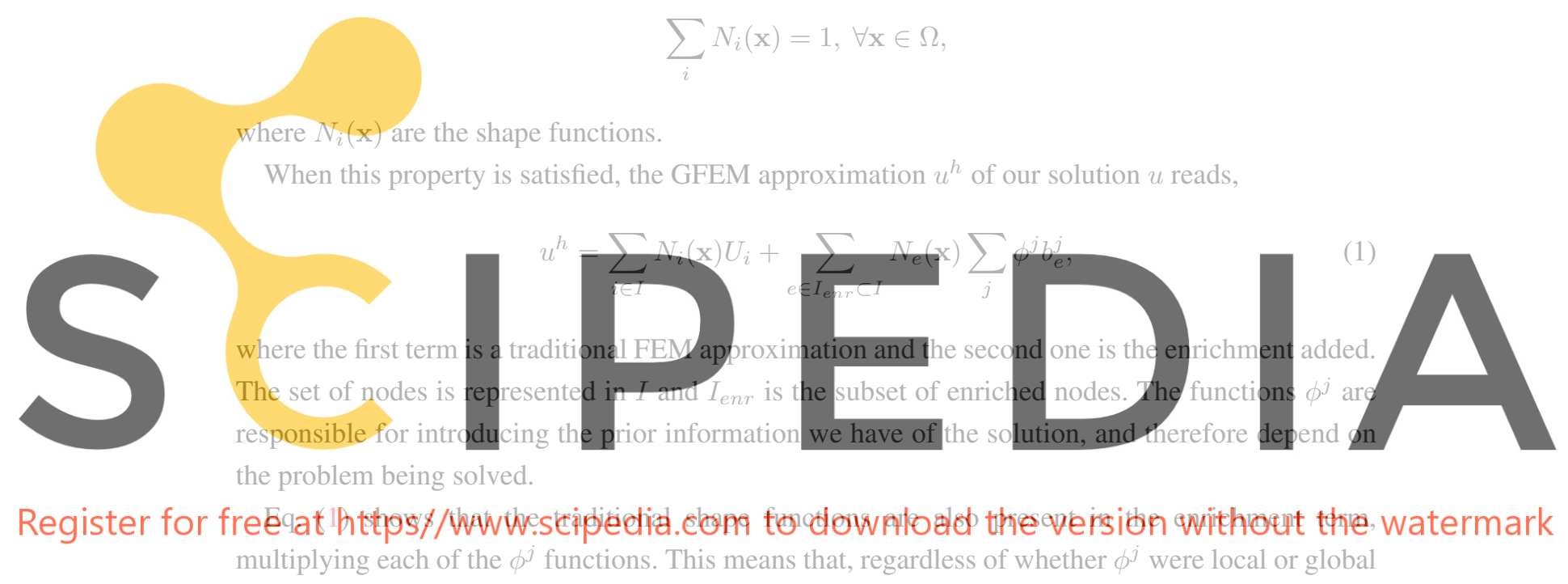

functions, each of the new shape functions $N_{e} \phi^{j}$ are local. In other words, the new shape functions

also have compact support. This property is vital for an easy and efficient implementation of the method [21].

The enriched nodes, the subset $I_{e n r}$ in Eq. (1), are just located in areas of interest where a greater accuracy is desired. These zones may be those where strong gradients occur or there are certain localized phenomena, for example cracks or damage. Thus, we can expect that the number of enriched nodes is relatively small compared to the total number of nodes of the discretization.

Moreover, GFEM has a very interesting meshless character which extends its applicability. Since the local features of a solution can be described in terms of nodal values at the nodes of the original mesh, neither remeshing nor structured meshes are needed. This is the case of welding processes. With a traditional $h$-adaptive strategy, the computational mesh is refined in the welding locations. However, due to the deformations induced in the process or in the assembly with other pieces, these locations could change. Thus, the reference configuration is not useful anymore and the mesh should be recomputed. Using GFEM this issue is circumvented, since the enrichment can be added to the original mesh where necessary and no refinement is needed.

As a counterpart, the degrees of freedom (DOFs) of the problem will be those attached to the nodes of the discretization, $U_{i}$, plus those attached to each enriched node and each of the enrichment 
functions $b_{e}^{j}$. Moreover, GFEM will typically require the use of a large number of integration points [22]. This is a consequence of using enriched shape functions which are in general nonpolynomial and require accurate enough quadrature rules.

Thus, from the point of view of computational efficiency, for any given accuracy, it is desirable to use a coarse mesh using the fewest enriched nodes and enrichment functions possible. In the limit, the best GFEM method will use only one enrichment function with the best possible local approximation properties of the solution. This is, as we will see, the fundamental advantage of the V-GFEM.

\subsection{The functional enrichment issue}

The main difficulty of the construction of a GFEM solution is the selection of the set of enrichment functions. The superiority of the GFEM compared with a conventional FEM depends directly on the efficacy of the enrichment functions chosen for a given application. In the literature, the following approaches can be found:
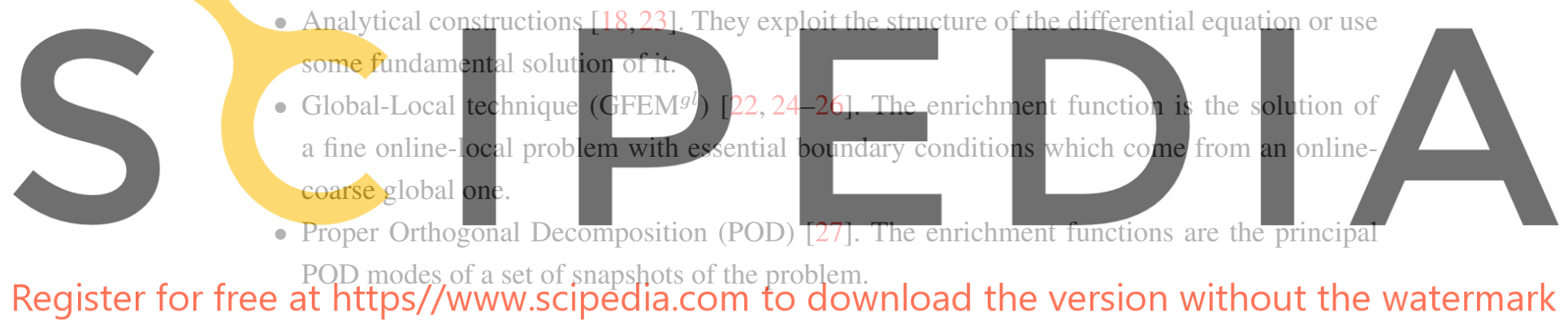

Analytical enrichment functions are problematic since they are hardly available for most applications of practical interest. Because of this, the construction of enriched functions by computational methods (Global-Local and POD) has become more relevant in recent years. The Global-Local implies solving iteratively both an online-global problem (coarse) and a online-local one (fine problem). Lastly, construction by POD has two drawbacks: firstly, the reduced basis indeed cannot in general capture all the details related to the solutions of models different from the one from which the reduced basis has been extracted [28] and secondly, the number of relevant modes could be very large causing GFEM to be too expensive.

When the problem is time dependent, the issue of selecting shape functions is complicated further. In the literature, one can find analytical time-dependent enrichment functions, but they are for very specific applications [22]. With a Global-Local approach it is possible to build time dependent enrichment functions, but the iterative process must be repeated at each time step. Lastly, with a POD approach the time-dependent could be potentially numerous.

Our work aims at overcoming these difficulties by proposing the use of a Computational Vademecum (also known as Computational Handbook) à la PGD for generating an improved approximation space. That is, an online-global and off-line local enrichment procedure is proposed. We believe that this approach has noteworthy advantages over existing methods. 


\section{VADEMECUM-GFEM}

The method proposed in this paper, the V-GFEM, is composed of two main elements: the GFEM as the framework, described in the previous section, and the vademecum as the key ingredient for constructing off-line the optimal enrichment function.

It is important to note that, in the V-GFEM, the computational vademecum is used to construct a better approximation space, not the solution in any subdomain. The GFEM framework provides many advantages since neither remeshing nor conformal meshes are needed, as is usually the case for Domain Decomposition techniques. Moreover, to construct the vademecum some phenomena can be neglected, such as transient effects, since they are accounted for in the global problem. This fact is in accordance with the results presented in [25].

In this section we elaborate the construction of the latter, which constitutes a true precomputed adaptive enrichment. Moreover, a general scheme of the V-GFEM is presented, providing further details of its implementation.

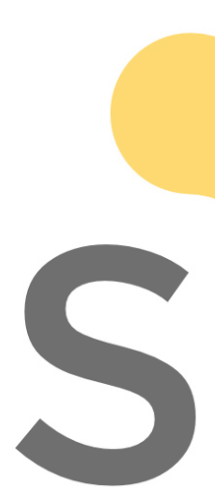
r

\subsection{Introducing a precomputed adaptive enrichment using the PGD}

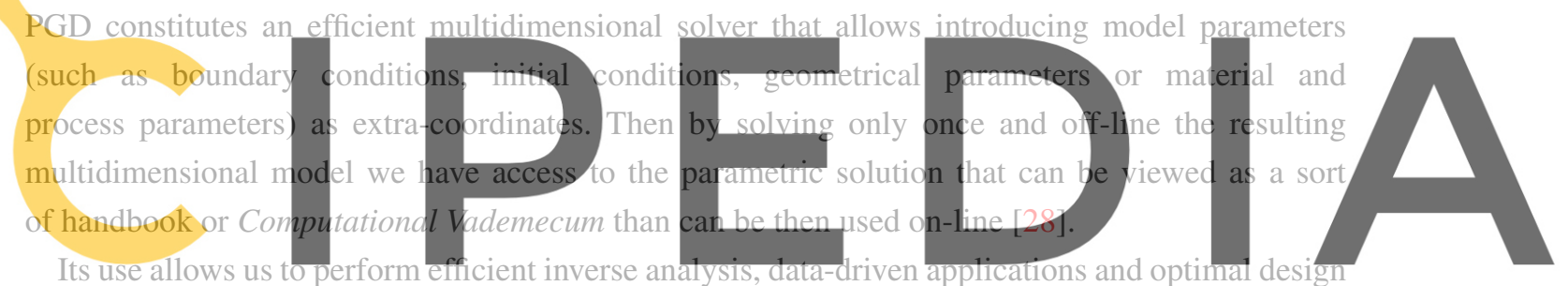

in large parametrical spaces [5]. Unlike other MOR approaches, PGD does not assume the form of Register for free at https/Www scipedia, com to download the version without the watermark

the fly.

In the V-GFEM, the computational vademecum is used to generate the proper enrichment function that depends on technological and material parameters of the process which are treated as extracoordinates.

Let us assume the mathematical model related to a certain physics. In general, this model will be expressed in terms of a system of partial differential equations (PDEs), defined in a spatial domain under given boundary conditions. The variational formulation of one PDE of this generic problem reads,

$$
\mathcal{B}\left(u, v ; p_{1}, p_{2}, \ldots, p_{n}\right)=\mathcal{L}\left(v ; q_{1}, q_{2}, \ldots, q_{m}\right),
$$

where $\mathbf{u}$ and $\mathbf{v}$ are the trial and test functions respectively and $p_{i}$ and $q_{j}$, with $i=1, . ., n$ and $j=1, . ., m$ are parameters on which the problem depends. The functions $\mathbf{u}$ and $\mathbf{v}$ are defined in the appropriate functional spaces [29].

Using traditional numerical methods (FEM, finite differences, spectral methods), the solution $\mathbf{u}$ of the variational form (2) is an approximation $u^{h}\left(\mathbf{x} ; \hat{p_{1}}, \hat{p_{2}}, \cdots, \hat{p_{n}}, \hat{q_{1}}, \hat{q_{2}}, \cdots, \hat{q_{m}}\right)$ for each particularization $\hat{p}_{i}, \hat{p_{j}}$ of the parameters $p_{i}$ and $q_{j}$. Then, if one wants to explore the parametrical space to obtain optimal solutions, the solution process must be repeated for any possible combination of particularizations of the parameters. If the number of parameters is large, this 
strategy becomes very costly, if not impossible. This limitation is often referred to in the literature as the "curse of dimensionality" [30].

Surrogate models consider the problem solution for some choices of the parameters and then use appropriate interpolation (e.g. Kriging). POD or RB-based techniques circumvent this difficulty by generating a reduced approximation basis from some solution snapshots after extracting the relevant information.

The PGD, on the other hand, constructs the approximated solution as follows,

$$
u^{h}=\sum_{k=1}^{N} F^{k}(\mathbf{x}) \prod_{i} P_{i}^{k}\left(p_{i}\right) \prod_{j} Q_{j}^{k}\left(q_{j}\right),
$$

where the parameters appear explicitly in the solution as extra-coordinates. Even if the problem becomes highly multidimensional in most cases the use of separated representations allows circumenting the aforementioned "curse of dimensionality". Thus, once the parametric solution is computed, the PGD solution provides all the possible solutions to the problem within the parametric domain $p_{i} \in\left[p_{i}^{\min }, p_{i}^{\max }\right], q_{j} \in\left[q_{j}^{\min }, q_{j}^{\max }\right]$. It is worth highlighting that the construction of the vademecum does not rely on the linearity of the problem. It can be also obtained in cases involving non-linear materials with state variables. However, the number of required modes, $N$, may depend on the complexity of the problem. For many applications of interest, tens of them are often enough.
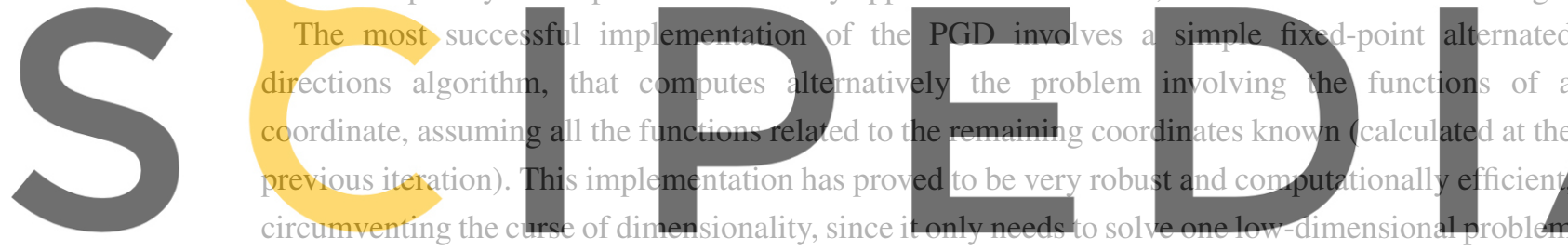

at a time. The interested reader can consult [28] to find all the details or Appendix A that summarizes

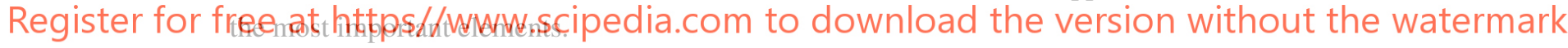

In the V-GFEM, the computational vademecum is introduced in a GFEM framework, which provides a performance that goes beyond the proposals made so far. This method is able to generate real-time customized approximation spaces, which accounts not only for material and technological parameters, but also for the value of the variable on the boundaries of the enriched region. This means that the enrichment region can "see" the changes that may occur in the global problem and adapt accordingly. All these parameters can vary during the simulation without loss of applicability and without compromising the solver efficiency.

The V-GFEM formulation can be expressed in the following compact form:

$$
u^{h}=\sum_{i \in I} N_{i}(\mathbf{x}) U_{i}+\sum_{e \in I_{e n r} \subset I} N_{e}(\mathbf{x}) \phi\left(\mathbf{x}, p_{1}, \ldots, p_{n}, q_{1}, \ldots, q_{m}\right) b_{e},
$$

where the enrichment function $\phi$, is nothing but a parametric general solution computed à la PGD. One can observe that, the trial space now depends explicitly of the parameters introduced in the vademecum.

The selection of these parameters should be done wisely, and should not be limited to technological parameters. Others, such as the values of the main variable at the border of the enriched region, $u_{\Gamma e}$ are of great interest in order to build a good enrichment function [22]. In 
this case, $u_{\Gamma e}$ should be represented in a global basis respect to the domain of the vademecum. This basis can be, for example, a polynomial one. In $2 \mathrm{D}$, for each side of the domain $u_{\Gamma e}$ reads,

$$
u_{\Gamma e}^{i}=a_{1}+a_{2} s_{i}+a_{3} s_{i}^{2}+\ldots+a_{n} s_{i}^{n-1},
$$

where $s^{i}$ is the local coordinate and $u_{\Gamma e}^{i}$ the value of $u_{\Gamma e}$ on the $i$-side. The uniqueness of $u_{\Gamma e}$ must be enforced at the corners of the domain.

The set of enriched nodes $I_{e n r}$ is a subset of the nodal set of the discretized problem. This set is determined by a geometric region (the so-called enrichment region) which is generally much smaller than the global domain. In the following examples, the enrichment region is attached to the movement of a heat source, where the thermal gradients are important and the solution cannot be found accurately using a coarse mesh.

\subsection{General scheme and implementation}

For the sake of clarity, let us assume a thermal transient problem with a moving heat source $s(\mathbf{x}, t)$ in a domain $\Omega$ and in the temporal interval $\mathcal{T} \in(0, T)$,
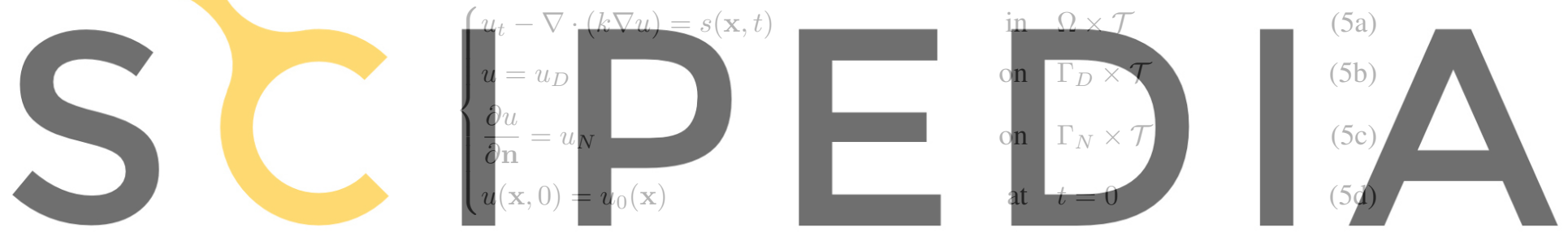

where the main variable $u$ is the temperature, $k$ the thermal diffusivity, the essential and natural

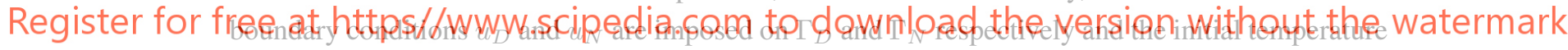
is $u_{0}$.

Attached to the source, an enrichment region, $\Omega_{e}$, is set. Its size must be determined for each particular case, taking into account that it should be large enough to capture the finer features of the solution. The geometry of this moving region is fixed and, at each time step, the nodes located inside are affected with the optimal enrichment function that simply consist in particularizing the parametric solution (computational vademecum) precomputed off-line. In short, in this region the trial space is more suitable to approximate the solution than the traditional FEM.

The V-GFEM can be divided into two stages: off-line and on-line stages, as is presented in Fig. 1.

In the off-line stage, the vademecum is computed introducing the technological parameters of the process, $\mathbf{p}=\left[p_{1}, p_{2}, \cdots, p_{n}\right]$, and the essential boundary conditions, $u_{\Gamma e}$, as extra-coordinates. The size of the domain of the computational vademecum is equivalent to the enriched region and a very fine discretization can be used because it is computed off-line. The model to solve is the same as in the original problem, but considering a moving reference frame $\Omega_{l}$. Thus, in this case, the movement of the heat source is considered from a convective operator and the problem reads

$$
\mathbf{v} \cdot \nabla \phi\left(x_{l}\right)-\nabla \cdot\left(k \nabla \phi\left(x_{l}\right)\right)=s\left(\mathbf{x}_{\mathbf{l}}\right) \quad \text { in } \quad \Omega_{l},
$$

where the main variable $\phi\left(x_{l}\right)$ is the enrichment function of the global problem. 


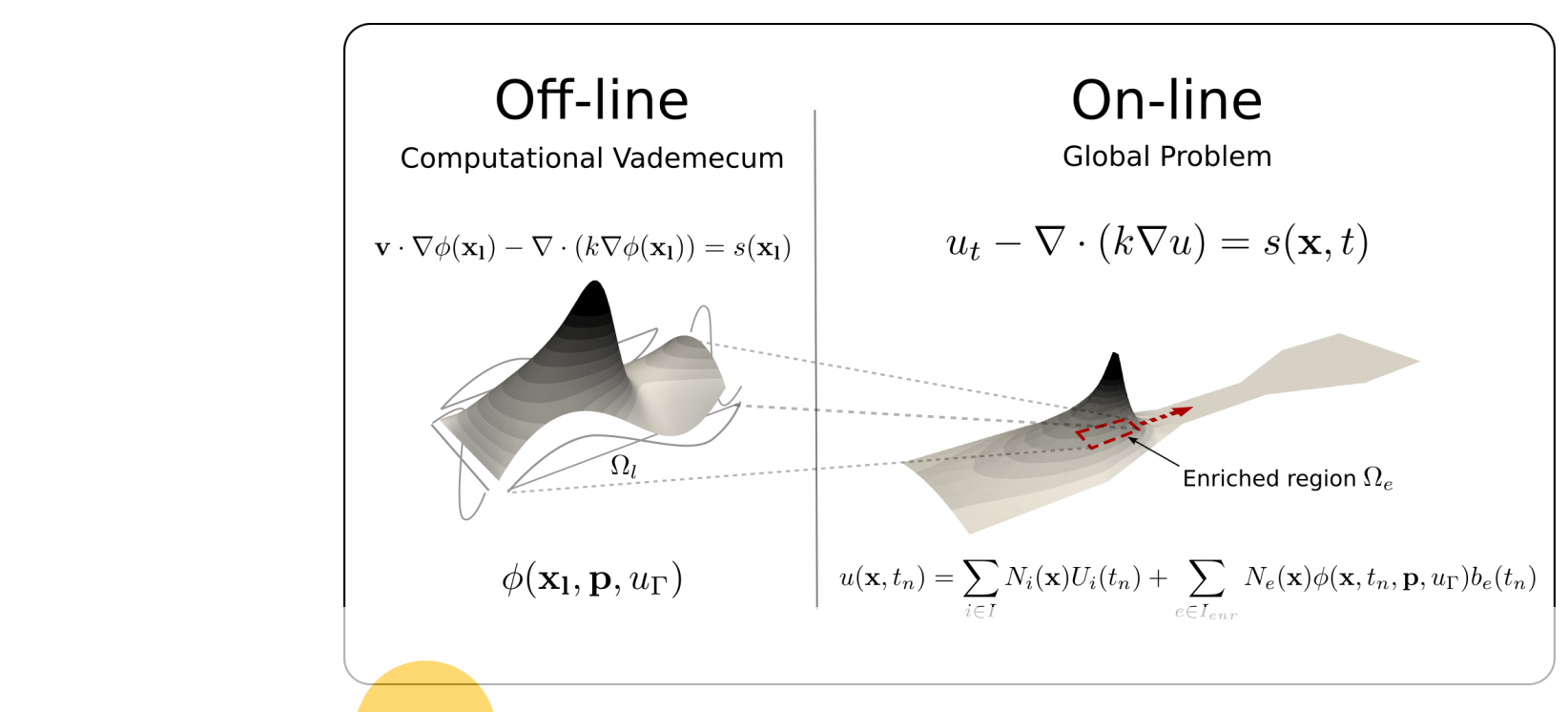

Figure 1. V-GFEM stages

Supposing a rectilinear motion, at each time $t, \Omega_{e}$ can be defined as $\Omega_{e}=\left\{\mathrm{x} \mid \mathrm{x}-\mathrm{v} t-\mathrm{r}_{0} \in \Omega_{l}\right\}$, where $r_{0}$ is the position vector between the vademecum reference system and $\Omega_{e}$ at initial time.
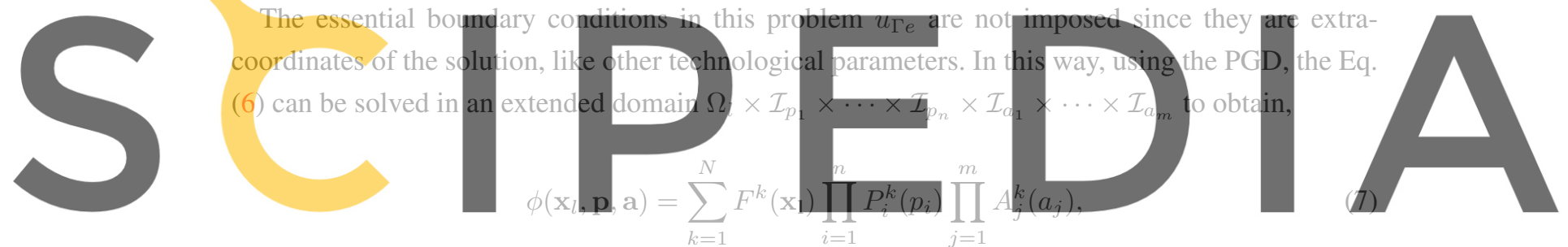

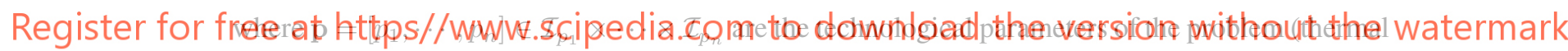
source velocity, power and shape of the heat source...) and $\mathrm{a}=\left[a_{1}, \ldots, a_{m}\right] \in \mathcal{I}_{a_{1}} \times \ldots \times \mathcal{I}_{a_{m}}$ are the coefficients of the polynomial basis of $u_{\Gamma e}$ in Eq. (4).

It is important to note that the computational vademecum generates steady enrichment functions, suitable when a stationary regime in the global problem is reached. Being rigorous, that means that to introduce the best approximation space in the pure transient phases of the simulation, the acceleration of the source should also be included as an extra-coordinate. The interested reader can consult [31] for more details about the this methodology. However, in most practical applications, the presented approach is enough.

In the on-line phase, at the time step $t$, the global problem is solved within the GFEM framework with the enrichment function obtained from particularizing the parametric local problem. This can be carried out in two ways:

- Explicitly: The particularization is carried out according to the technological parameters at the current time step but the solution at the enrichment domain boundary at the previous time step, i.e. $\phi^{t}\left(\mathbf{p}^{t}, u_{\Gamma e}^{t-1}\right)$. This explicit scheme works well if the time step is not very large and the solution evolves smoothly as is the case, for example, in welding simulation.

- Implicitly: Using a point-fixed strategy, the global problem is solved iteratively updating $u_{\Gamma e}$ until the enrichment function $\phi^{t}$ converges. This scheme is presented in Fig. 2, where $\hat{\phi}$ is an 
auxiliary function to check this convergence and the upper index $t-1$ represents a value at the previous time step of the current one.

It should be noted that, the explicit strategy is a particularization of the implicit one where $\hat{\phi}$ is not computed, omitting the decision block. For the examples presented here, the explicit scheme provides excellent results.

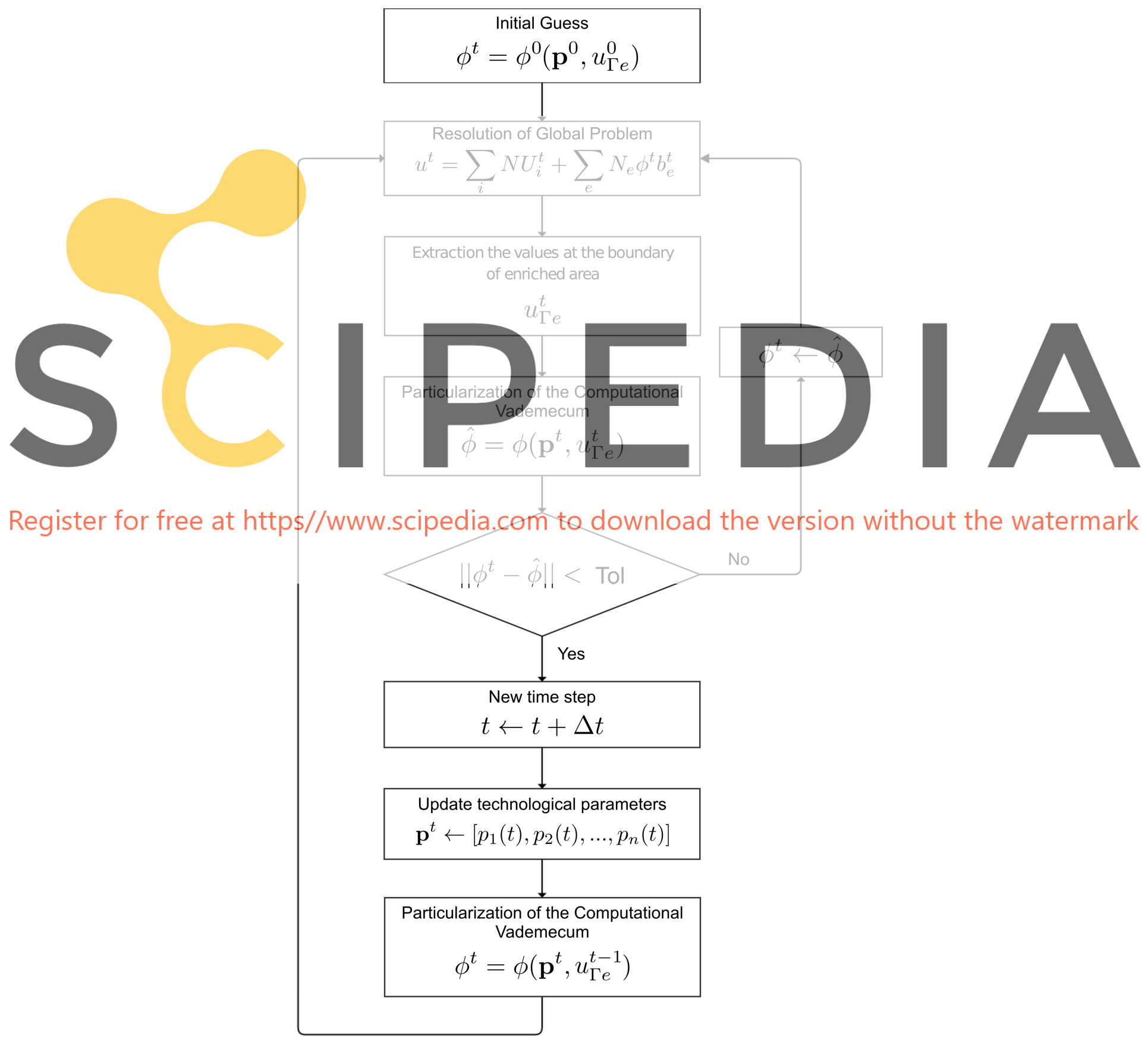

Figure 2. Online stage of V-GFEM 
At each time step, a good initial guess is the enrichment function from the boundary conditions in the parametric solution coming from the previous time step. For stationary problems, a simple and effective idea is to introduce the particularization of the vademecum with $u_{\Gamma e}=0$, a sort of "fundamental solution" of the problem, performing the fixed-point iterations if necessary. Note that this point-fixed iteration does not imply a great computational effort, since the updating of the enrichment function is performed at negligible cost. In addition, to solve the global problem, because the domain remains fixed, several reanalysis techniques can be used, alleviating the computational cost of the solution [22].

This strategy is analogous to the iterative strategy concerned with improving the local boundary conditions in the $\mathrm{GFEM}^{g l}$ presented in [24, 25] and analyzed in [32]. In these references an alternative strategy is proposed, the introduction of a buffer zone. This zone enlarges the local domain using layers of coarse elements in order to introduce smoother boundary conditions to the local problem. In the context of the V-GFEM, this implies changing the domain of the computational vademecum with no other alterations to the general scheme.

In order to extract $u_{\Gamma e}^{t}$ at each time step, the global solution $u^{t}$ has to be projected at the borders of the enriched area in the basis given by (4). In this work a simple $L_{2}$ projection is carried out.

It should be noticed that, depending on the values of the simulation parameters, inside the enriched region, the enriched solution in a coarse element could be approximated by the coarse shape functions, leading to a rank deficiency. Two techniques could be introduced to avoid this rank deficiency: a perturbation method [33], in which all the nodes are still enriched, and the off-line computation of a new vademecum which gives the reduced set of nodes that must be enriched. The latter solution not only circumvents the issue, but also reduces the number of enriched nodes increasing the efficiency of the V-GFEM. In the Appendix B the details of this technique can be found.

\section{NUMERICAL EXAMPLES}

As it was said in the introduction, the V-GFEM is particularly well suited for the simulation of processes involving moving heat sources, such as welding or surface heat treatments. This is because there is a small region where the physical changes are much more pronounced (Heat Affected Zone, HAZ) and the process strategy should be optimized in large parametric spaces.

In this section, several numerical examples using the V-GFEM for an unsteady heat equation are shown. This equation is encountered in the thermal analysis of the mentioned processes [2]. The examples presented in this section are relatively simple with the purpose of illustrating the potential of the V-GFEM for these kind of simulations: the domain is 2D and the technological and material parameters do not correspond to any real process. However, the method can be applied to the 3D simulation of real industrial processes. 


\subsection{Statement of the problem}

Let us consider the transient PDE (5) in the domain $\Omega$ depicted in Fig. 3, where $s(\mathbf{x}, t)$ reads

$$
s(\mathbf{x}, t)=\frac{Q}{\sigma \sqrt{2 \pi}} \exp \left(\frac{1}{2}\left(\frac{x-\left(x_{c}+V t\right)}{\sigma}\right)^{2}-\frac{1}{2}\left(\frac{y-y_{c}}{\sigma}\right)^{2}\right),
$$

where $\mathbf{x}=(x, y), Q$ controls the power of the heat source and $\sigma$ is related with the size of the area of incidence of the heat source. $V$ is the magnitude of the velocity $\mathbf{v}=V \mathbf{u}_{x}$ where $\mathbf{u}_{x}$ is the unitary vector of the coordinate $x$. The starting point of the heat source is $\left(x_{c}, y_{c}\right)$.

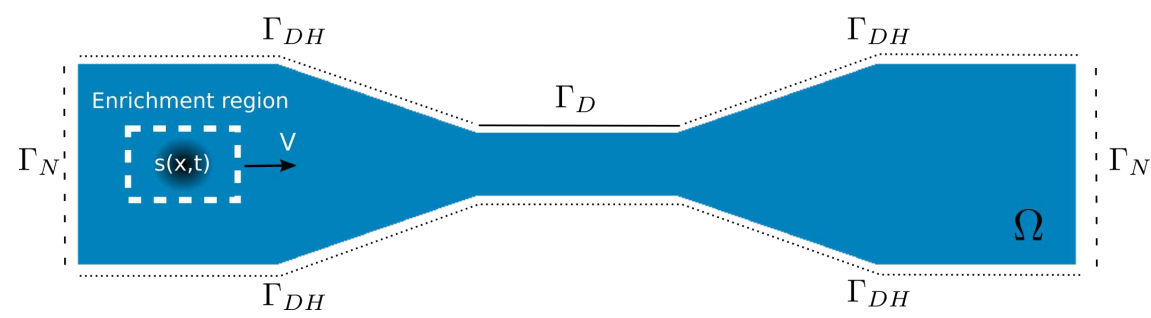

Figure 3. Scheme of the problem

Let us assume homogeneous Dirichlet boundary conditions on $\Gamma_{D H}$ and homogeneous Neumann boundary conditions on $\Gamma_{N}$. On $\Gamma_{D}$ a Dirichlet boundary condition is imposed and its value depends on the example. The coefficient of diffusivity takes the value 1 and the other parameters will vary depending on the example.

To perform the V-GFEM, an enrichment region, $\Omega_{e}$, is defined attached to the heat source, as can be seen in Fig. 3. This region, a subdomain of the global domain, moves with the source whilst retaining its shape and size. Then, in an off-line stage, the computational vademecum for this problem is computed in a moving reference frame. Moreover, the velocity of this reference frame is considered as an extra-coordinate.

In this example, the next parameters were introduced in the vademecum as extra-coordinates: the power of the heat source $Q$, the magnitude of the thermal source velocity $V$, the region of incidence of the heat source described by $\sigma$ and the temperature at the boundary of the enrichment region $u_{\Gamma e}$. To introduce the latter a polynomial basis of order three was used. Thus, the parametric enrichment function reads:

$$
\phi=\phi\left(\mathbf{x}, Q, V, \sigma, a_{1}, \cdots, a_{12}\right)=\sum_{k=1}^{N} F^{k}(\mathbf{x}) G_{i}^{k}(Q) H_{i}^{k}(V) I_{i}^{k}(\sigma) \prod_{j=1}^{12} A_{j}^{k}\left(a_{j}\right) .
$$

In Fig. 4, the computational vademecum constructed for this example is presented. The visualization can be easily done with the Paraview open source plugin developed in our research group [34].

Then, the variational formulation of the problem (5) is constructed,

$$
\left(u^{t+1 *}, \frac{u^{t+1}}{\Delta t}\right)+\mathbf{a}\left(u^{t+1 *}, u^{t+1}\right)=\left(u^{t+1 *}, \frac{u^{t}}{\Delta t}\right)+\left(u^{t+1 *}, s^{t+1}\right)
$$

where a Galerkin spatial discretization and an implicit $\alpha$-method, with $\alpha=1$, as a temporal scheme are used. For clarity, the argument of the functions have been omitted. 

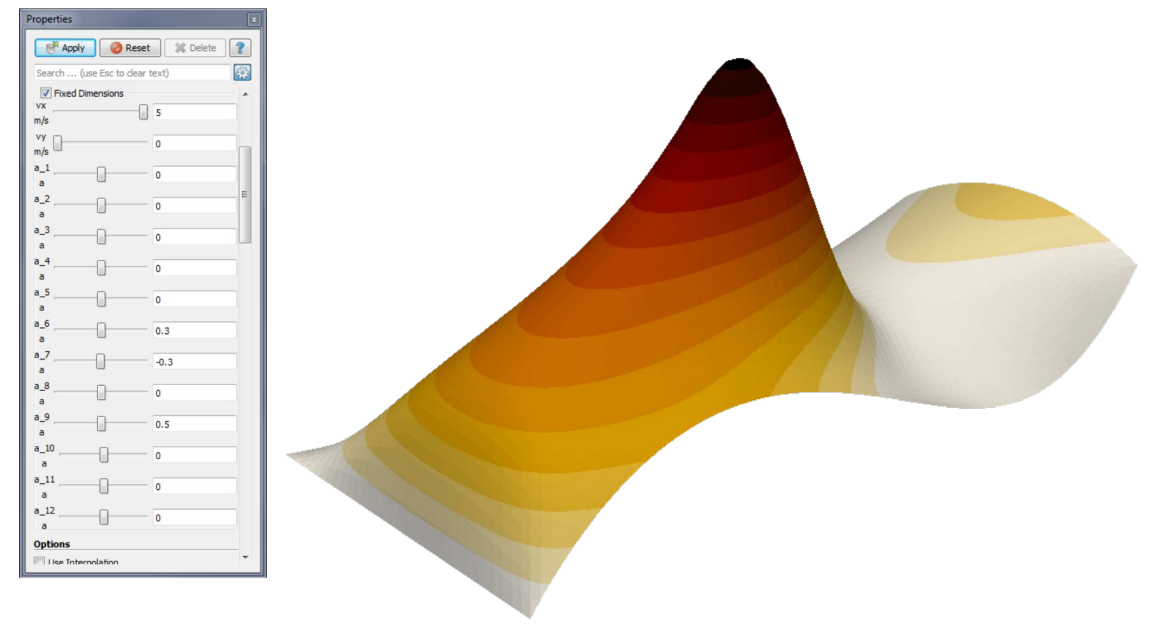

Figure 4. Computational Vademecum

In Eq. (8), $\mathbf{a}\left(u^{t+1 *}, u^{t+1}\right)$ is the Laplacian operator and the trial and test functions, $u^{t+1}$ and $u^{t+1^{*}}$ respectively, are constructed using the approximation given by the V-GFEM in Eq. (3). It is important to realize that, exactly as in the GFEM $^{g l}[24,25]$, in the V-GFEM the enrichment function is time dependent. Thus, the operator $\left(u^{t+1 *}, \frac{u^{t}}{\Delta t}\right)$ on the right hand side should be carefully computed since it involves two functions, $u^{t+1 *}$ and $u^{t}$ which are represented using different approximation spaces. For this purpose, two particularizations of the vademecum must be stored when using this temporal integration scheme.

In this example, to perform the numerical integration, the coarse mesh equipped with a large number of integration points (of the order of 100) at the enriched elements has been used. The number of points has been determined by numerical tests as in [24]. Advanced integration strategies, taking into account the off-line/in-line nature of the presented work are still in progress.

In the next section we will analyze the solution of this problem solved with the V-GFEM formulation under different scenarios. The computational vademecum, computed off-line, is the same and the algorithm described in Fig. 2 was followed in all of these cases.

\subsection{V-GFEM VS FEM}

Let us start illustrating the important improvement that can be achieved with an enriched trial space in comparison with a traditional FEM space. In this example the following boundary conditions and technological parameters were selected: $Q=10 \mathrm{~W} / \mathrm{m}^{2}, V=1 \mathrm{~m} / \mathrm{s}, \sigma=\sqrt{0.05}$ and $u=0$ on $\Gamma_{D}$.

The V-GFEM and the FEM simulations were carried out using the same coarse mesh depicted in Fig. 5. For clarity, the temperature field is represented as a relief map. As can be seen in Fig. 5, the V-GFEM solution, in solid color, significantly improves the FEM solution, wire-frame represented, at the vicinity of the heat source. Differences up to $30 \%$ in the maximum temperature value were observed.

Here, the enrichment function is trivially updated, since the technological parameters are constant and the temperature field at the border of the enriched region does not change significantly during the 


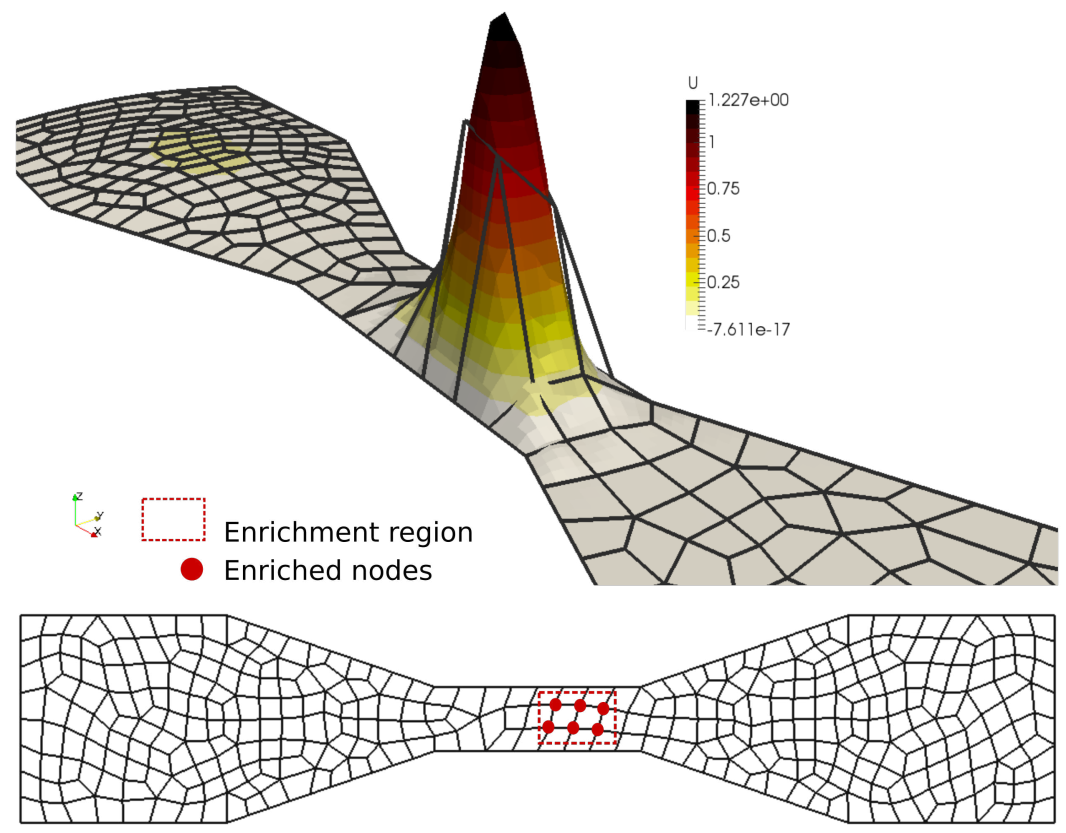

Figure 5. V-GFEM solution vs FEM solution

simulation. Thus, in this case, the advantage of having a computational vademecum is the possibility of performing just one calculation for a given family of problems.

Adaptation to the boundary conditions Let us now consider different boundary conditions of the global problem, setting $u=1$ on $\Gamma_{D}$ in the upper part of the narrow zone. Then, when the source enters in this narrow region, the global essential boundary conditions affects the optimal trial space. With the V-GFEM, due to the fact that the enrichment function is an explicit function of $u_{\Gamma e}$, the best approximation space is always achieved. In Fig. 6 one can observe how the vademecum generates different enrichment functions $\phi$ at different instants during the simulations according to the global boundary conditions.

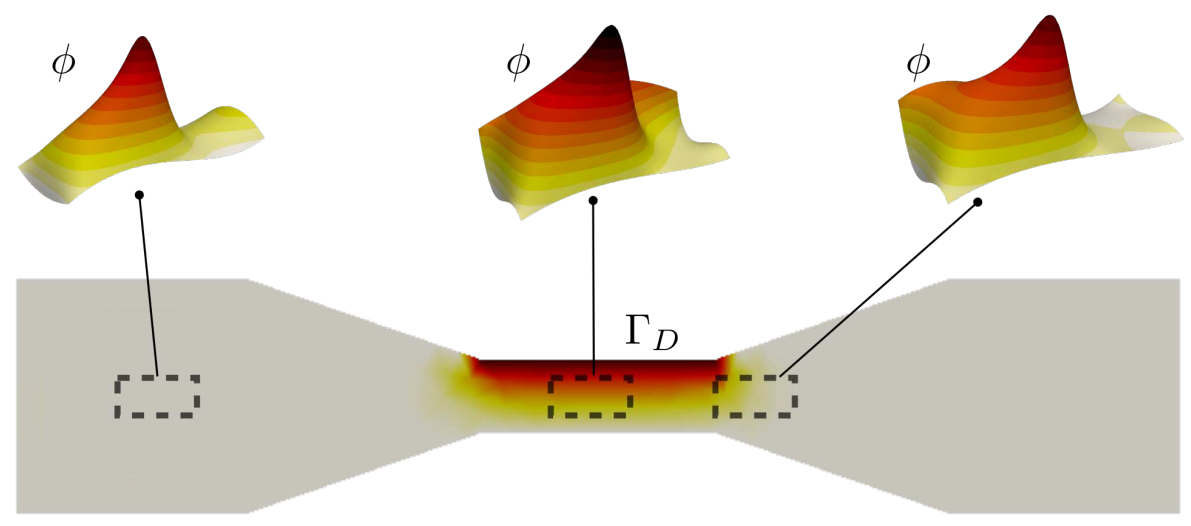

Figure 6. Adaptivity of the enrichment function 
This allows us to obtain a considerably improved solution with respect to the FEM one using the same coarse mesh, as is shown in Fig. 7. The V-GFEM solution, in wire-frame, is equipped with the optimal trial space at the current time step. In solid color, the FEM solution using the same coarse mesh is represented. If the enrichment function is not updated as is indicated in Fig. 6, the improvement obtained with the V-GFEM will be reduced by $15 \%-20 \%$ as we will see in the following sections.

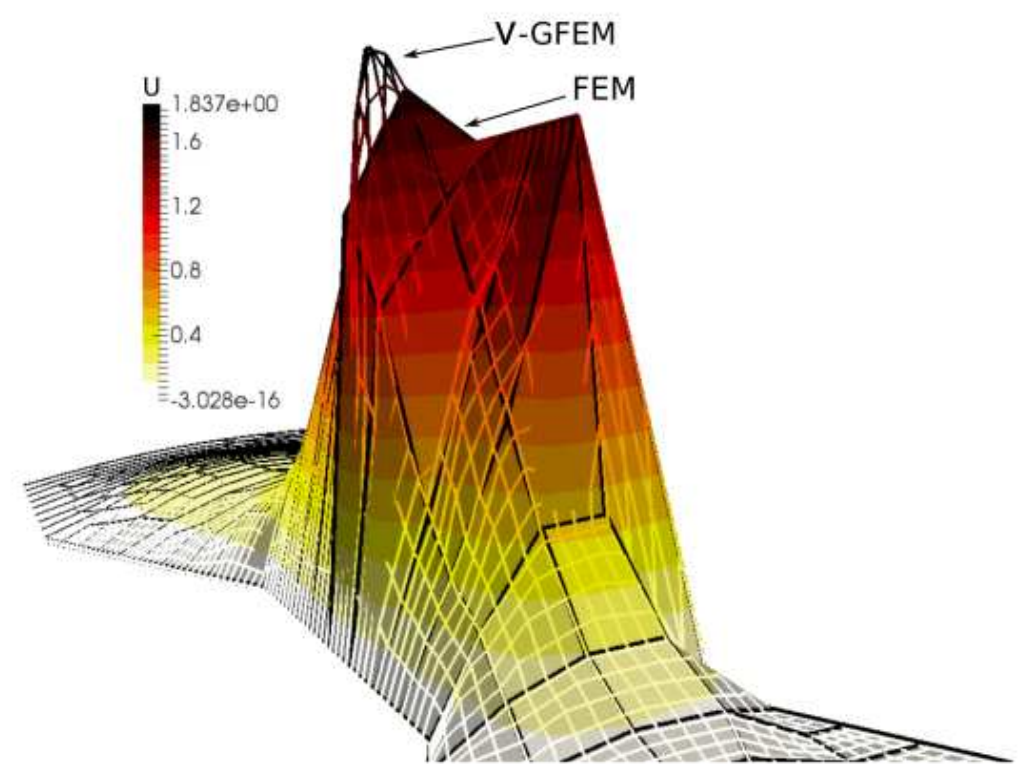

Figure 7. V-GFEM solution vs FEM solution with change in BCs

\subsection{Accounting for variable technological parameters}

We move forward now to analyze the key feature of the V-GFEM: the adaptation in real time to changes in the technological parameters of the simulation.

Let us consider an thermal source velocity which changes from $30 \mathrm{~m} / \mathrm{s}$ to $5 \mathrm{~m} / \mathrm{s}$ at a certain point during the process. The other technological parameters take the fixed values $Q=10 \mathrm{~W} / \mathrm{m}^{2}$ and $\sigma=\sqrt{0.025}$. All the Dirichlet and Neumann BCs are homogeneous.

In Fig. 8, the two enrichment functions generated by the vademecum at the two different velocities are shown. The function $\phi_{1}$ was particularized at $30 \mathrm{~m} / \mathrm{s}$ and the function $\phi_{2}$ at $5 \mathrm{~m} / \mathrm{s}$.

Then, two simulations were carried out, in the first one, we chose $\phi=\phi_{1}$ and we keep it constant during the complete simulation. In the second one, on the other hand, $\phi$ was updated conveniently to the thermal source velocity change. In Fig. 9 the relative errors of both of the simulations are presented. It can be seen that updating the enrichment function diminishes the maximum relative error from $27 \%$ to $5 \%$ with respect to a reference solution (a FEM solution with a very fine mesh).

Let us now see another example. In this case, is the area of incidence of the heat source, controlled by the parameter $\sigma$, which changes during the simulation. The essential and natural boundary conditions are homogeneous again. We set the other technological parameters, $Q=10 \mathrm{~W} / \mathrm{m}^{2}$, $V=10 \mathrm{~m} / \mathrm{s}$. We started the simulation with $\sigma=\sqrt{0.05}$ and at a certain point it changes to 

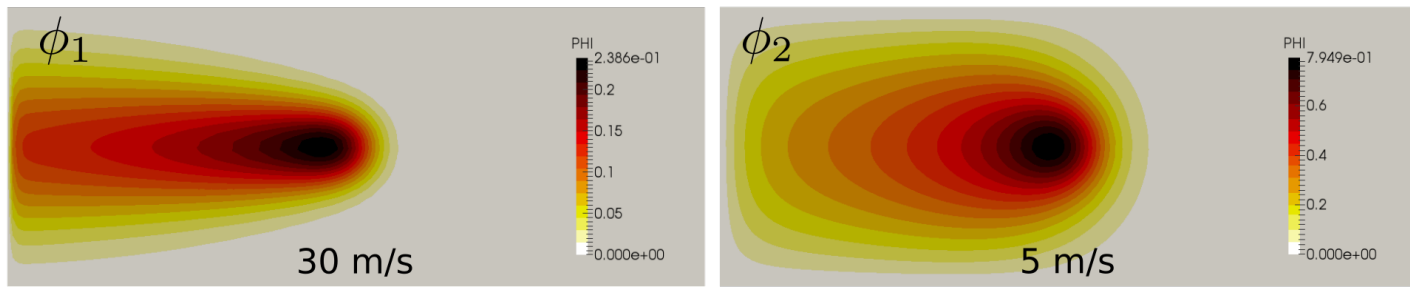

Figure 8. Enrichment functions at different advance velocities
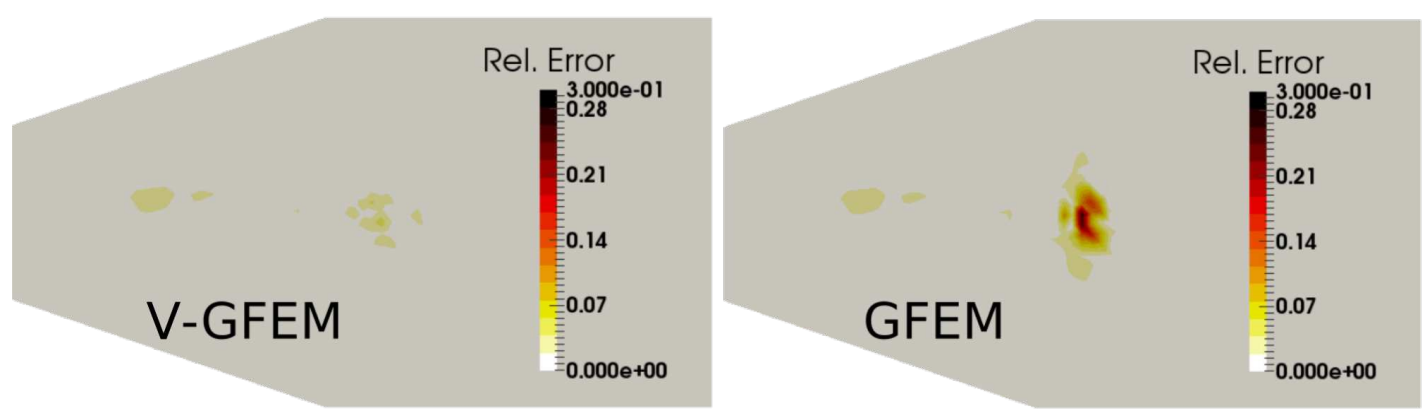

Figure 9. Relative errors with and without adapting the enrichment function

$\sigma=\sqrt{0.005}$. The two enriched functions generated by the computational vademecum at those instants are $\phi_{1}$ and $\phi_{2}$ respectively as can be seen in Fig. 10.
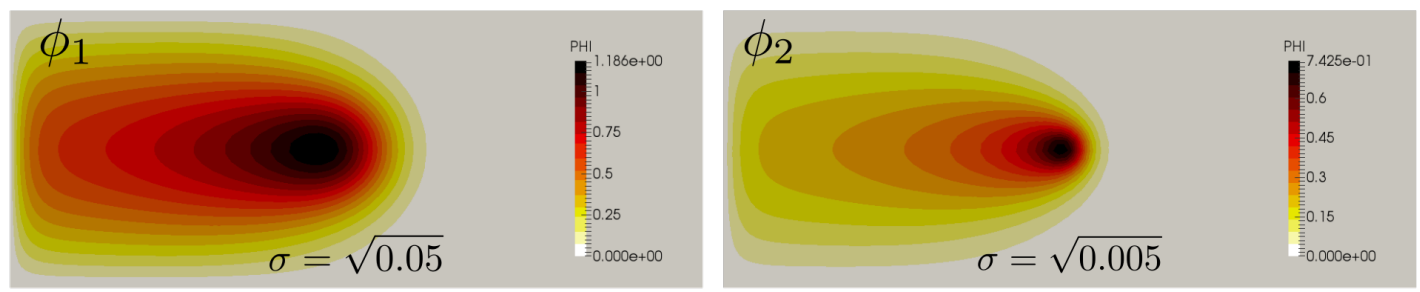

Figure 10. Enrichment functions at different areas of incidence of the heat source

As before, we performed two simulations. In the first one, we set $\phi=\phi_{1}$ and we keept it constant during the entire simulation. In the second one, $\phi$ was updated conveniently, adapting it to the change of area of incidence. In this case, the fact of updating or not the enrichment function results in a difference of $25 \%$ in maximum temperature with respect to the reference solution, as can be observed in Fig. 11. In this figure, the reference FEM solution is in wire-frame and in solid color the solution without updating the enrichment function and by updating it within the V-GFEM framework.

In the last example, one can see that the thermal history of a given point could be significantly affected if an adaptation of the enrichment fuction is not carried out. If a GFEM without updating is considered, the maximum temperature in the point drops around a $25 \%$ respect to the reference solution. Conversely, if the V-GFEM is used, the results are in very good agreement with the reference solution as can be observed in Fig. 12. It should be noted that, since updating the vademecum has a negligible cost, this improvement is obtained with no additional computational effort. 

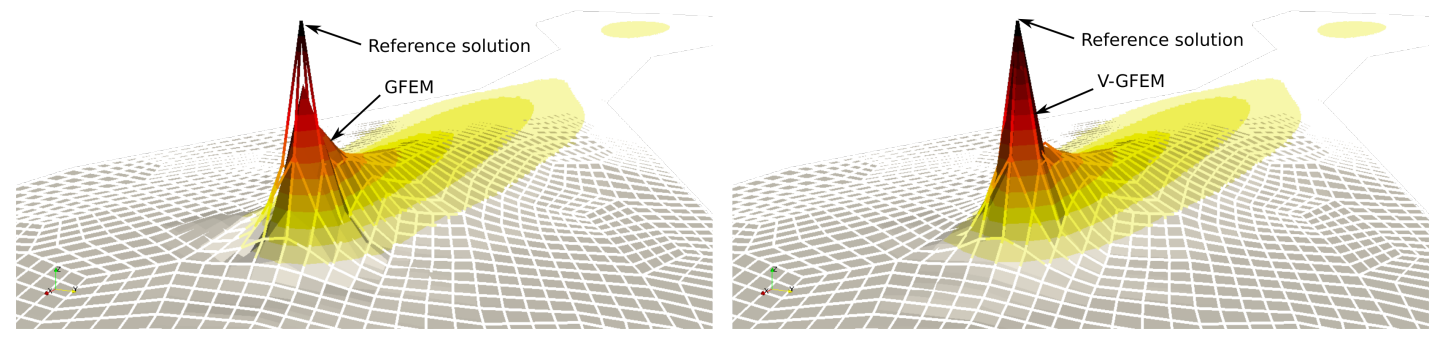

Figure 11. Difference between solution with and without updating the enrichment function

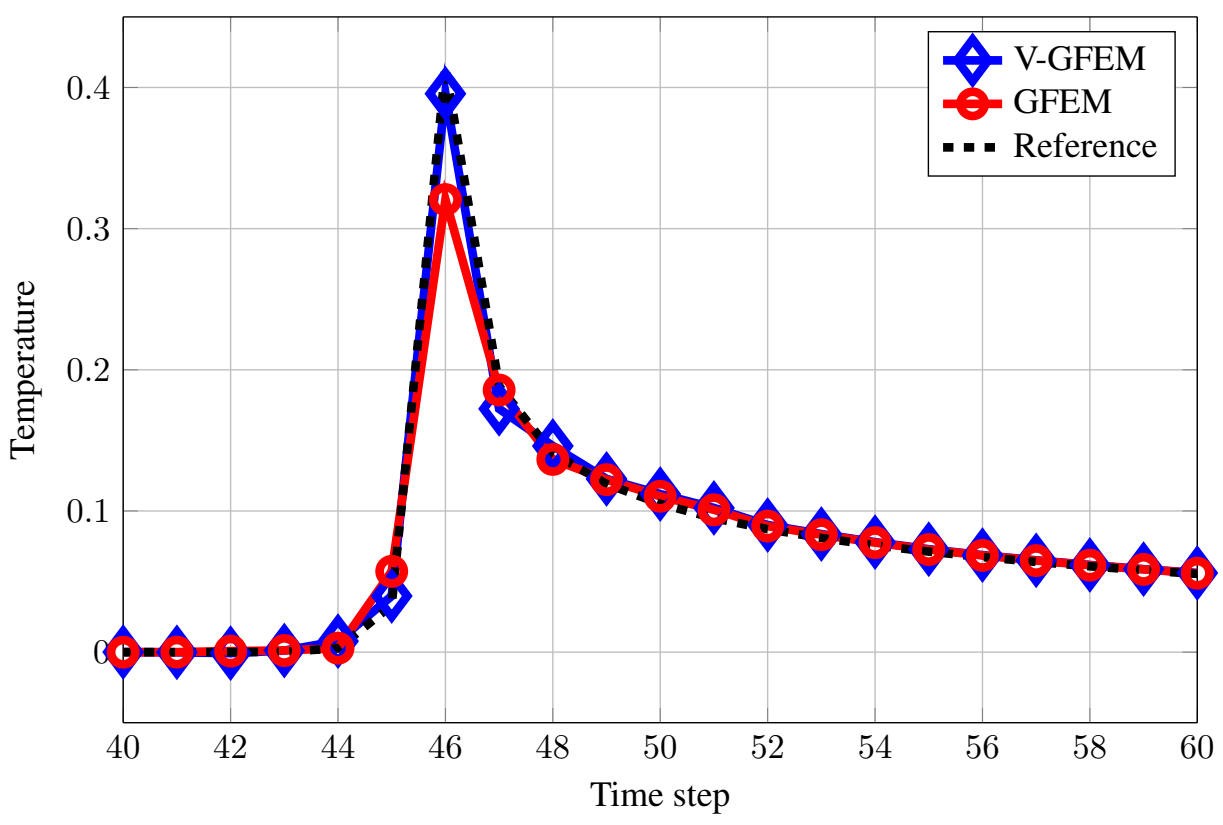

Figure 12. Thermal history of a material point

In simulation of welding, the thermal analysis is usually coupled with a metallurgical model to obtain the changes in phases and in the microstructure of the material. For this analysis, a thermal history with errors of the order of $25 \%$ may represent in practice obtaining a completely wrong numerical prediction of mechanical properties.

\subsection{Timing}

In order to obtain a measure of the relative computational savings we obtain with the just presented technique, we solved the problems with a sequence of FEM meshes refined by bisection (i.e., each mesh contained four times the number of degrees of freedom of its parent mesh.)

Our conclusion is that, in general, the proposed V-GFEM technique could obtain the same level of accuracy of its equivalent FEM mesh, but refined twice more. The resulting CPU savings turns out to be of one order of magnitude.

We strongly believe, nevertheless, that the advantages to be obtained in three-dimensional meshes will be even more important. Moreover, due to the fact that the enrichment function is known 
(computed off-line), advanced integration strategies, as in [35], can be used to significantly reduce the number of integration points with the consequent saving in computational time.

Their analysis constitutes our current effort of research and will be published elsewhere.

\section{CONCLUSIONS AND PERSPECTIVES}

In this paper, a new numerical method, the V-GFEM, for efficient simulations of manufacturing processes has been presented. Its main advantage consists in adapting the trial space in real-time to approximate the solution of the problem optimally. This is achieved thanks to the key ingredient of V-GFEM, the Computational Vademecum, which is computed off-line. Thus, it can be build in a very accurate way with no impact on the computational cost of the global simulation.

The V-GFEM, not only inherits the good features of the GFEM formulations such as local approximability, conforming and meshless character of the enrichment, but also brings an important advantage: it only needs one enrichment function which is the best possible for a given family of problems at any instant of the simulation.

The computational cost of the V-GFEM is the same as any other GFEM approach in which only one analytical enrichment function is used, for example in initial versions of XFEM [18]. In the examples presented here, in general, we have obtained a difference of an order of magnitude between the V-GFEM solution and FEM solution with a $h$-adapted mesh to obtain the same accuracy.

There could be a plethora of possible applications of the V-GFEM since it is a general framework as is GFEM. In particular, in future research, we will address the mechanical part of manufacturing processes with localized physics, as we have been done here for the thermal problem. In this case, the main challenge will be the construction of a vademecum taking into account internal variables which depend on the thermomechanial history of the material considering elasto-plastic models.

\section{ACKNOWLEDGMENTS}

The authors wish to thank the ESI Group for its financial support through the ESI group - ECN Chair. In addition, E. Cueto and D. González gratefully acknowledge the financial support by the Spanish Ministry of Economy and Competitiveness through Grant number CICYT DPI2014-51844-C2-1-R, and by the Regional Government of Aragon and the European Social Fund.

\section{A. CONSTRUCTION OF A COMPUTATIONAL VADEMECUM $\grave{A} L A$ PGD}

In this annex the construction of a computational vademecum for a V-GFEM strategy is elaborated. Without losing generality, a one-dimensional problem similar to the benchmarks presented in [24] is used. Let us consider the following transient equation defined in $\Omega=(0, L)$ and in the time interval $\mathcal{T}=(0, T)$, 


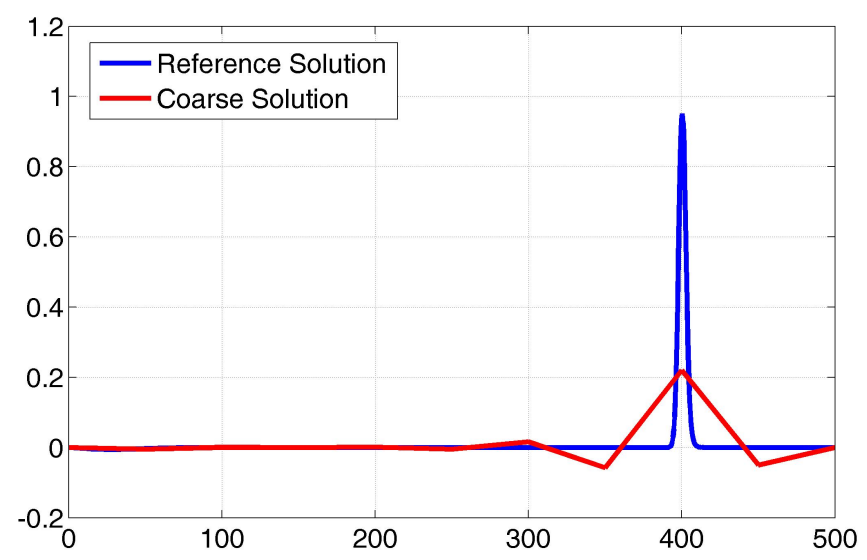

Figure 13. Reference and coarse solutions for the 1D example

$$
\left\{\begin{array}{l}
\frac{\partial u}{\partial t}-\frac{\partial^{2} u}{\partial x^{2}}=Q(x, t) \\
u(0, t)=u(0, L)=0 \\
u(x, 0)=0
\end{array}\right.
$$

with the following moving source:

$$
\begin{aligned}
Q(x, t)=2 \gamma\left(x-\left(x_{0}+v t\right)\right) \exp \left(-\gamma\left(x-\left(x_{0}+v t\right)\right)^{2}\right) & \\
-4 \gamma^{2}\left(x-\left(x_{0}+v t\right)\right)^{2} \exp \left(-\gamma\left(x-\left(x_{0}+v t\right)\right)^{2}\right) & \\
& +2 \gamma \exp \left(-\gamma\left(x-\left(x_{0}+v t\right)\right)^{2}\right) .
\end{aligned}
$$

In the above problem $L=500, T=500, x_{0}=0, v$ is the advance velocity of the source and $\gamma$ is a parameter which controls the shape of the source.

The solution of this problem presents a localized zone with high gradients, which travels with the same advance velocity $v$ and cannot be captured with a coarse mesh. In Fig. 13 the reference solution and the solution obtained with a coarse mesh with a constant size of elements of $L / 50$ for $v=1$ and $\gamma=0.1$ at $t=400$ is presented. The coarse approximation space is not able to represent the solution even if sufficient quadrature rule is used. In order to apply the V-GFEM, a moving subdomain $\Omega_{l}=(\hat{x}-\delta, \hat{x}+\delta)$ attached to the source is defined, where $\hat{x}=x_{0}+v t$ is the middle point and $\delta$ the half length. Then, the same physics but in a moving reference frame should be solved:

$$
\left\{\begin{array}{l}
-v \frac{\partial \phi}{\partial x_{l}}-\frac{\partial^{2} \phi}{\partial x_{l}^{2}}=Q_{l}\left(x_{l}\right) \\
\phi(-\delta)=\phi(\delta)=0 .
\end{array}\right.
$$


where the source, in this reference frame, reads:

$$
\begin{aligned}
Q_{l}\left(x_{l}\right)=2 \gamma\left(x_{l}-\hat{x}\right) \exp \left(-\gamma\left(x_{l}-\hat{x}\right)^{2}\right) & \\
& -4 \gamma^{2}\left(x_{l}-\hat{x}\right)^{2} \exp \left(-\gamma\left(x_{l}-\hat{x}\right)^{2}\right)+2 \gamma \exp \left(-\gamma\left(x_{l}-\hat{x}\right)^{2}\right) .
\end{aligned}
$$

The half length $\delta$ should be large enough to correctly integrate the localized source. In this example $\delta=50$ has been chosen. In order to precompute the solution to the former problem for a family of different heat sources and different advance velocities, one can consider both parameters as extra-coordinates, $v \in \mathcal{I}_{v}$ and $\gamma \in \mathcal{I}_{\gamma}$. Then, in an extended domain $\left(x_{l}, v, \gamma\right)=\Omega_{l} \times \mathcal{I}_{v} \times \mathcal{I}_{\gamma}$, the PGD can be used to construct the solution in the following form,

$$
\phi\left(x_{l}, v, \gamma\right) \approx \sum_{i=1}^{N} X_{i}\left(x_{l}\right) \cdot V_{i}(v) \cdot G_{i}(\gamma) .
$$

For that purpose we consider the weighted residual form related to Eq. (11)

$$
\int_{\Omega_{l} \times \mathcal{I}_{v} \times \mathcal{I}_{\gamma}} \phi^{*} \cdot\left(-v \frac{\partial \phi}{\partial x_{l}}-\frac{\partial^{2} \phi}{\partial x_{l}^{2}}-Q_{l}\right) \mathrm{d} x_{l} \cdot \mathrm{d} v \cdot \mathrm{d} \gamma=0 .
$$

At iteration $n<N$ the solution $\phi^{n}\left(x_{l}, v, \gamma\right)$ reads

$$
\phi^{n}\left(x_{l}, v, \gamma\right)=\sum_{i=1}^{n} X_{i}\left(x_{l}\right) \cdot V_{i}(v) \cdot G_{i}(\gamma)
$$

and the new trial function $\phi^{n+1}\left(x_{l}, v, \gamma\right)$ is searched according to

$$
\phi^{n+1}\left(x_{l}, v, \gamma\right)=\sum_{i=1}^{n+1} X_{i}\left(x_{l}\right) \cdot V_{i}(v) \cdot G_{i}(\gamma)=\phi^{n}\left(x_{l}, v, \gamma\right)+X_{n+1}\left(x_{l}\right) \cdot V_{n+1}(v) \cdot G_{n+1}(\gamma)
$$

with the test function $\phi^{\star}$ given by

$$
\begin{aligned}
\phi^{\star}\left(x_{l}, v, \gamma\right)=X^{\star}\left(x_{l}\right) \cdot V_{n+1}(v) \cdot G_{n+1}(\gamma)+ & \\
& +X_{n+1}\left(x_{l}\right) \cdot V^{\star}(v) \cdot G_{n+1}(\gamma)+X_{n+1}\left(x_{l}\right) \cdot V_{n+1}(v) \cdot G^{\star}(\gamma) .
\end{aligned}
$$

By introducing the trial and test functions, Eqs. (16) and (17) respectively, into the weak form, Eq. (14), and using an appropriate linearisation, functions $X_{n+1}\left(x_{l}\right), V_{n+1}(v)$ and $G_{n+1}(\gamma)$ are calculated. When considering the simplest linearisation strategy, the alternated direction fixed point algorithm, the following steps are repeated until reaching convergence:

1. With $V_{n+1}^{(r-1)}(v)$ and $G_{n+1}^{(r-1)}(\gamma)$ given at the previous iteration of the non linear solver $(r-1)$ (arbitrarily initialized at the first iteration: $V_{n+1}^{(0)}(v)$ and $G_{n+1}^{(0)}(\gamma)$ ), all the integrals in $\mathcal{I}_{v} \times \mathcal{I}_{\gamma}$ are performed, leading to a boundary value problem involving $X_{n+1}^{(r)}\left(x_{l}\right)$.

2. With $X_{n+1}^{(r)}\left(x_{l}\right)$ just calculated and $G_{n+1}^{(r-1)}(\gamma)$ given at the previous iteration of the non linear solver $(r-1)$, all the integrals in $\Omega \times \mathcal{I}_{\gamma}$ are performed, leading to an algebraic problem involving $V_{n+1}^{(r)}(v)$. 
3. With $X_{n+1}^{(r)}\left(x_{l}\right)$ and $V_{n+1}^{(r)}(v)$ just updated, all the integrals in $\Omega \times \mathcal{I}_{v}$ are performed, leading to an algebraic problem involving $G_{n+1}^{(r)}(\gamma)$.

4. The convergence is checked by calculating

$$
\begin{aligned}
\mathcal{E}^{r}=\| X_{n+1}^{(r)}\left(x_{l}\right)-X_{n+1}^{(r-1)}( & \left.x_{l}\right) \| \\
& +\left\|V_{n+1}^{(r)}(v)-V_{n+1}^{(r-1)}(v)\right\|+\left\|G_{n+1}^{(r)}(\gamma)-G_{n+1}^{(r-1)}(\gamma)\right\| .
\end{aligned}
$$

When $\mathcal{E}^{r}$ becomes small enough the just computed functions are incorporated into the approximation of the solution:

$$
\left\{\begin{array}{l}
X_{n+1}\left(x_{l}\right)=X_{n+1}^{(r)}\left(x_{l}\right) \\
V_{n+1}(v)=V_{n+1}^{(r)}(v) \\
G_{n+1}(\gamma)=G_{n+1}^{(r)}(\gamma) .
\end{array}\right.
$$

The convergence of the enrichment iteration is checked as soon as the non-linear iteration converges, by evaluating the norm of the just computed term $\left\|X_{n+1}(\mathbf{x}) \cdot T_{n+1}(t) \cdot K_{n+1}(k)\right\|$; the residual norm, or any appropriate error estimator based on quantities of interest [36].

In Fig. 14, some particularizations of the vademecum of this simple example are shown.
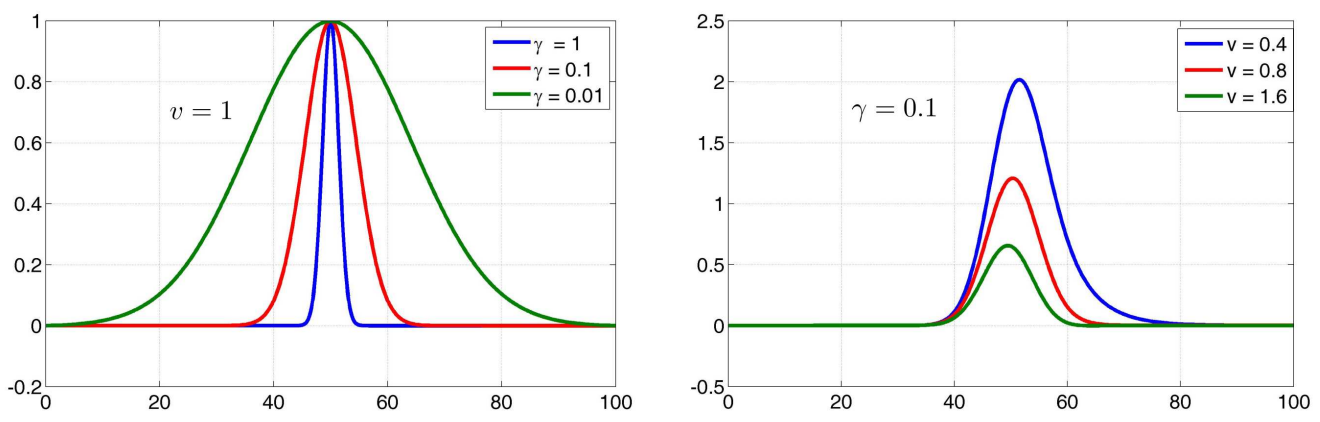

Figure 14. Particularizations of the vademecum

This computational vademecum can be used to enrich the coarse finite element space as presented in this work. Theoretical results about errors and convergence of GFEM global-local methods can be found in [17,32].

\section{B. CIRCUMVENTING THE RANK DEFICIENCY}

For certain values of the parameters the parametric solution in certain regions of its domain of definition could be accurately represented by the subjacent FE coarse approximation, leading to rank deficiency. This fact indicates that as soon as the enriched solution in a particular region of the domain in which the enrichment is performed can be accurately described by the coarse approximation, the associated nodes should be removed from the enrichment nodal list $I_{e}$. For this purpose we generate a second vademecum, by projecting, again offline, the parametric solution, $\phi\left(\mathbf{x}, Q, V, \sigma, u_{\Gamma e}\right)$, from which the enrichment function is extracted, on different coarse FE element 
approximations associated with different mesh size $h \in\left[h_{1}, \cdots, h_{M}\right]$. This projection writes

$$
\int_{\Omega} u^{h_{i} *} u^{h_{i}} \mathrm{~d} \omega=\int_{\Omega} u^{h_{i} *} \phi \mathrm{d} \omega
$$

with $u^{h_{i}}\left(\mathbf{x}, Q, V, \sigma, u_{\Gamma e}\right)$ the projected solution on a mesh of size $h_{i}$, and then the projection must be performed for each considered mesh characterized by its size $h_{i}$, even if the mesh size could be introduced as an extra-coordinate within the PGD framework. Then, as soon the projected parametric solutions $u^{h_{i}}$ are available, the parametric residual,

$$
r\left(\mathbf{x}, Q, V, \sigma, u_{\Gamma e}\right)=\phi-u^{h_{i}}
$$

could be computed and compared to a threshold value $\epsilon$ according to

$$
r^{2}<\epsilon^{2}
$$

As soon as for the given parameters the parametric solution constituting the GFEM enrichment is particularized, at each node in the enrichment region (domain in which the enrichment solution id defined) we particularize the residual (20), and if the inequality (21) holds, we remove from the enrichment nodal list that node. This procedure ensure an optimal enrichment while avoids rank deficiency.

\section{REFERENCES}

1. President's Information Technology Advisory Committee. Computational science: Ensuring America's competitiveness. Report to the President, 2005.

2. J.M. Bergheau and R. Fortunier. Finite Element Simulation of Heat Transfer. ISTE. Wiley, 2013.

3. G. Rozza, D.B.P. Huynh, and A.T. Patera Reduced basis approximation and a posteriori error estimation for affinely parametrized elliptic coercive partial differential equations application to transport and continuum mechanics. Archives of Computational Methods in Engineering, 15:229-275, 2008.

4. A. Cohen, and R. DeVore. Approximation of high-dimensional parametric PDEs. Acta Numerica, 24:1-159, 2015.

5. F. Chinesta, A. Leygue, F. Bordeu, J. V. Aguado, E. Cueto, D. González, I. Alfaro, A. Ammar, and A. Huerta. PGD-Based Computational Vademecum for Efficient Design, Optimization and Control. Archives of Computational Methods in Engineering, 20(1):31-59, 2013.

6. F. Chinesta, A. Leygue, B. Bognet, Ch. Ghnatios, F. Poulhaon, F. Bordeu, A. Barasinski, A. Poitou, S. Chatel, and S. Maison-Le-Poec. First steps towards an advanced simulation of composites manufacturing by automated tape placement. International journal of material forming, 7(1):81-92, 2014.

7. J. Fish and T. Belytschko. A First Course in Finite Elements. John Wiley \& Sons, 2007.

8. J. Fish. The s-version of the finite element method. Computers \& Structures, 43(3):539 - 547, 1992.

9. J. Fish and Z. Yuan. Multiscale enrichment based on partition of unity. International Journal for Numerical Methods in Engineering, 62(10):1341-1359, 2005.

10. A. Ammar, E. Pruliere, J. Férec, F. Chinesta, and E. Cueto. Coupling finite elements and reduced approximation bases. European Journal of Computational Mechanics/Revue Européenne de Mécanique Numérique, 18(5-6):445463, 2009.

11. A. Ammar, F. Chinesta, and E. Cueto. Coupling finite elements and proper generalized decompositions. International Journal for Multiscale Computational Engineering, 9(1), 2011.

12. S. Niroomandi, I. Alfaro, D. Gonzalez, E. Cueto, and F. Chinesta. Real-time simulation of surgery by reduced-order modeling and x-fem techniques. International journal for numerical methods in biomedical engineering, 28(5):574$588,2012$.

13. J.M. Melenk. On Generalized Finite Element Methods. PhD thesis, University of Maryland, 1995. 
14. I. Babuška and J. M. Melenk. The partition of unity method. International Journal for Numerical Methods in Engineering, 40(4):727-758, 1997.

15. I. Babuška, G. Caloz and J.E. Osborn. Special finite element methods for a class of second order elliptic problems with rough coefficients, SIAM Journal on Numerical Analysis, 31(4):945-981, 1994.

16. C.A. Duarte and J.T. Oden. Hp clouds-A meshless method to solve boundary-value problem. TICAM report, 95-05, 1995.

17. J.M. Melenk and I. Babuška. The partition of unity finite element method: Basic theory and applications. Computer Methods in Applied Mechanics and Engineering, 139(14):289 - 314, 1996.

18. J. Dolbow and T. Belytschko. A finite element method for crack growth without remeshing. Int. J. Numer. Meth. Eng, 46(1):131-150, 1999.

19. J.T. Oden, C.A. Duarte and O.C. Zienkiewicz. A new cloud-based hp finite element method. Computer Methods in Applied Mechanics and Engineering, 153(1):117-126, 1998.

20. T. Strouboulis, I. Babuška and K. Copps. The design and analysis of the generalized finite element method, Computer Methods in Applied Mechanics and Engineering, 181(1):43-69, 2000.

21. O.C. Zienkiewicz and R.L. Taylor. The finite element method for solid and structural mechanics. Butterworthheinemann, 2005.

22. P.J. O'Hara. A Multi-scale Generalized Finite Element Method for Sharp, Transient Thermal Gradients. PhD thesis, University of Illinois, 2010.

23. R. Merle and J. Dolbow. Solving thermal and phase change problems with the extended finite element method. Computational mechanics, 28(5):339-350, 2002.

24. P.J. O'Hara and C.A. Duarte and T. Eason and D.-J. Kim. Generalized Finite Element Analysis of ThreeDimensional Heat Transfer Problems Exhibiting Sharp Thermal Gradients. Computer Methods in Applied Mechanics and Engineering, 198(21-26):1857-1871, 2009.

25. P.J. O'Hara and C.A. Duarte and T. Eason. Transient Analysis of Sharp Thermal Gradients Using Coarse Generalized Finite Element Meshes. Computer Methods in Applied Mechanics and Engineering, 200(5-8):812-829, 2011.

26. C.A. Duarte and D-J. Kim. Analysis and applications of a generalized finite element method with global-local enrichment functions. Computer Methods in Applied Mechanics and Engineering, 197(6):487-504, 2008.

27. W. Aquino, J.C. Brigham, C.J. Earls, and N. Sukumar. Generalized finite element method using proper orthogonal decomposition. International journal for numerical methods in engineering, 79(7):887, 2009.

28. F. Chinesta and E. Cueto. PGD-Based Modeling of Materials, Structures and Processes. Springer International Publishing Switzerland, 2014.

29. C. Johnson. Numerical solution of partial differential equations by the finite element method. Courier Corporation, 2012.

30. R. Bellman. Dynamic Programming. Princeton University Press, 1957.

31. F. Chinesta, N. Bur, P. Joyot, Ch. Ghnatios, P. Villon, and E. Cueto. Advanced computational vademecums for automated fibre placement processes. In press.

32. V. Gupta, D.-J. Kim, and C.A. Duarte. Analysis and Improvements of Global-Local Enrichments for the Generalized Finite Element Method. Computer Methods in Applied Mechanics and Engineering, 245-246:47-62, 2012.

33. C.A. Duarte, I. Babuška, and J.T Oden. Generalized finite element methods for three-dimensional structural mechanics problems. Computers \& Structures, 77(2):215-232, 2000.

34. F. Bordeu. The paraviewpxdmfreader. https://rom.ec-nantes.fr/resources/separated-variables-representationvisualisation/.

35. F. Olmos, F. Chinesta, and R. Torres. Finite element resolution of convection-diffusion equations with interior and boundary layers. International journal for numerical methods in fluids, 22(7):643-671, 1996.

36. A. Ammar, F. Chinesta, P. Diez, and A. Huerta. An error estimator for separated representations of highly multidimensional models. Computer Methods in Applied Mechanics and Engineering, 199(25):1872-1880, 2010. 